\title{
An efficient Architecture for Multiband-MIMO with LTE- Advanced Receivers for UWB Communication Systems
}

\author{
Ahmed Qaiem $^{1}$, Dr. Anil Kumar ${ }^{2}$, Dr. Rajeev Paulus ${ }^{3}$ \\ ${ }^{I}$ (M.Tech Student, Department of Electronic and Communication/ Wireless Communication Engineering, \\ SHIAT, India) \\ ${ }^{2}$ (Assistant Professor, Department of Electronic and Communication, SHIAT, India) \\ ${ }^{3}$ (Assistant Professor, Department of Electronic and Communication, SHIAT, India)
}

\begin{abstract}
The emerging ultra wideband (UWB) system offers a great potential for the design of high speed short-range wireless communications. In order to satisfy the growing demand for higher data rates, one possible solution is to exploit both spatial and multipath diversities via the use of multiple-input multiple-output (MIMO) and proper coding techniques. In this paper, candidate architecture for LTE Advanced receiver is proposed. Based on the combination of MIMO techniques and flexible spectrum access, LTE-Advanced terminals will require the increasing of the analog front-end complexity. This is a general framework is proposed to analyze the performance of multiband UWB-MIMO systems regardless of specific coding schemes. Simulation and measurement results show that, in a Gaussian case, the bit error rate is similar when using the proposed architecture and the state of the art front-end stack-up structure. A complexity evaluation study reveals significantly reduced power consumption of the proposed single front-end architecture. Finally, simulation results are presented to support the theoretical analysis.
\end{abstract}

Keywords: LTE-Advanced, Double IQ structure, Code multiplexing structure. Ultra wideband (UWB), wireless personal area networks (WPANs).

\section{INTRODUCTION}

Ultra wideband (UWB) is an emerging technology that offers great promises to satisfy the growing demand for low cost and high-speed digital wireless home networks. UWB is defined as any radio transmission that occupies a bandwidth of more than $20 \%$ of its center frequency, or nominally more than $500 \mathrm{MHz}$. In 1998, the Federal Communications Commission (FCC) has mandated that UWB radio transmission can legally operate in the range from $3.1 \mathrm{GHz}$ to $10.6 \mathrm{GHz}$, at a transmit power of $\mathrm{dBm} / \mathrm{MHz}$ [1]. Depending on how the available bandwidth is utilized, UWB can be divided into two groups: single band and multiband. A traditional UWB technology is based on single-band systems employing carrier-free communications [2]-[4].

It is implemented by directly modulating information into a sequence of impulse-like waveforms, which occupy the available band width of $7.5 \mathrm{GHz}$. Multiple users, can be supported via the use of time hopping or direct sequence spreading approaches. The single band system faces a challenging problem in building RF and analog circuits, and in designing a low complexity receiver that can capture sufficient multipath energy. Recently, multiband UWB schemes were proposed in [5]-[6], in which the UWB frequency band is divided into several sub-bands. Each sub-band occupies a bandwidth of at least $500 \mathrm{MHz}$ in compliance with the FCC regulations. By interleaving the symbols across sub-bands, multiband UWB can maintain the transmit power as if the large $\mathrm{GHz}$ bandwidth is utilized. The advantage is that multiband approach allows the information to be processed over a much smaller bandwidth, thereby reducing overall design complexity as well as improving spectral flexibility and worldwide compliance.

Internet of things: this is the greatest challenge of mobile communications. All incoming standards need to provide higher throughputs in compact terminals. One can see that all proposed solutions converge towards the same techniques: OFDM, MIMO, adaptive coding and modulation, as well as scalable bandwidths. These techniques require an increasing complexity of terminals, leading to additional cost and power consumption. Particularly from the RF front-ends point of view, OFDM imposes high PAPR constraints and good linearity [7], MIMO conducts to the multiplication of RF chains, scalable bandwidth imposes wider bandwidth characteristics of RF components [8]. Recently, proposed several solutions meant to reduce complexity of multi-band [9] [10] and multi-antenna [11][12] front-ends. In this article looked forward merging these solutions in order to propose a novel architecture for simplified LTE-Advanced terminals. This matter is treated by the 3GPP [13] working groups that are studying the future mobile phone standards with very high data rates and mobility. In fact, in current propositions for LTE-Advanced PHY-layer, the use of combined multiple-antenna capabilities and multiple separated frequency channels are very seducing for the downlink transmission. Meanwhile the use of these combined techniques supposes high cost architectures for the user embedded terminal. 
The rest of the paper is organized as follows. In Section II, presented the multiband UWB-MIMO system model, including the signal modulation, channel model, receiver description, and Detection technique and summarize the main LTE-Advanced key points, focusing on multi-band and multi-antenna needs. In Sec. IV details simulated and measured performance. Finally, Conclusions and forthcoming works are drawn in Sec. V.

\section{SYSTEM DESIGN MULTIPLE UWB-MIMO SYSTEM MODEL}

Multi brand UWB-MIMO system model Consider a multiband OFDM scenario that has been proposed in the IEEE 802.15.3a WPAN standard [33]. The available UWB spectrum of $7.5 \mathrm{GHz}$ is divided into several sub-bands, each with bandwidth B.W of at least $500 \mathrm{MHz}$. Each user utilizes one sub-band per transmission. For each user, signals from all transmit antennas share the same sub-band. Within each sub-band, OFDM modulation with subcarriers issued at each transmit antenna. Different bit rates are achieved by using different channel coding, frequency spreading, or time spreading rates. A multiband UWB system considered with fast band-hopping rate, i.e., the signal is transmitted one frequency-band during one OFDM symbol interval, and then moved to a different frequency band at the next interval.

\section{A. Transmitter Description}

A peer-to-peer multiband UWB system utilized $\mathrm{N}_{\mathrm{t}}$ transmit and $\mathrm{R}_{\mathrm{t}}$ receive antennas, as shown in Fig. 1. The information is encoded across $\mathrm{N}_{\mathrm{t}}$ transmit antennas, Subcarriers, and K OFDM blocks .At the transmitter, the coded information sequence from a channel encoder is partitioned into blocks of $\mathrm{N}_{\mathrm{b}}$ bits. Each blocks

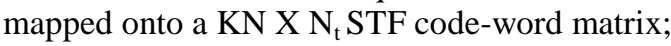

$$
\mathrm{D}=\left[\begin{array}{llll}
\mathrm{D}_{0}^{T} & \mathrm{D}_{1}^{T} & \cdots & \mathrm{D}_{K-1}^{T}
\end{array}\right]^{T}
$$

Where

$$
\mathrm{D}_{k}=\left[\begin{array}{llll}
\mathrm{d}_{1}^{k} & \mathrm{~d}_{2}^{k} & \cdots & \mathrm{d}_{N_{t}}^{k}
\end{array}\right],
$$

In which

$\mathbf{d}_{i}^{k}=\left[d_{i}^{k}(0) d_{i}^{k}(1) \cdots d_{i}^{k}(N-1)\right]^{T}$ for $i=1,2, \ldots$,

The symbol, represents the complex symbol to be transmitted over subcarrier by transmit antenna during the MIMO symbol period. The matrix is normalized to have average energy, where stands for the expectation operation, and denotes the Frobenius norm. At the System block, the transmitter applies -point IFFT over each column of the matrix, yielding an OFDM symbol of length. In order to mitigate the effect of inter symbol interference, a cyclic prefix of length is added to the output of the IFFT processor.

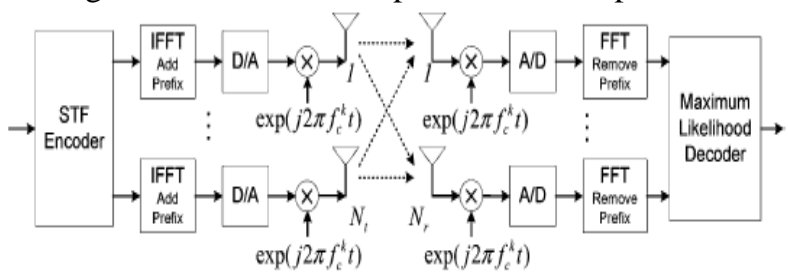

Figure-1 Multiband UWB-MIMO system.

\section{B. Channel Model}

Tap-delay line Nakagami-M fading channel model with L taps. At the K the OFDM block, the channel impulse response from the $\mathrm{i}$ transmit antenna to the $\mathrm{j}$ receive antenna can be described as;

$$
h_{i j}^{k}(t)=\sum_{l=0}^{L-1} \alpha_{i j}^{k}(l) \delta\left(t-\tau_{l}\right)
$$

The measurements in UWB channels indicate that the amplitude of each path follows either a log-normal or Nakagami- $m$ distribution. The Nakagami fading parameter, $m$, describes the severity of the fading. It can be any real value that satisfies $m>=1 / 2$. The smaller them, the more severe the fading, with $m=1$ and $m=\infty$ corresponding to the Rayleigh fading and nonfading channel, respectively. The advantage of Nakagami-m statistics is that they can model a wide range of fading conditions by adjusting their fading parameters. In fact, Nakagami-m distributions with large value are similar to the log-normal distributions. Therefore, performing the theoretical analysis based on the Nakagami- m distribution. 


\section{Receiver Processing}

The signal received at each receive antenna is a superposition of $\mathrm{N}_{t}$ the transmitted signals corrupted by additive white Gaussian noise. Assume that the receiver perfectly synchronizes to the band switching pattern. The received RF signal at each receive antenna is down converted to a complex baseband signal, matched to the pulse waveform, and then sampled before passing through an OFDM demodulator. After the OFDM modulator discards the cyclic prefix and performs an N-point FFT, maximum-likelihood detection is jointly performed across all $\mathrm{N}_{\mathrm{T}}$ receive antennas. The choice of prefix length greater is than the duration of the channel impulse response. Assuming that the receiver has a perfect knowledge of the channel state information, while the transmitter has no channel information. The receiver exploits a maximum likelihood decoder; where the decoding process is jointly performed on $\mathrm{N}_{\mathrm{T}}$ receive signal vectors. The decision rule can be stated as;

$$
\hat{\mathbf{D}}=\arg \min _{\mathbf{D}} \sum_{j=1}^{N_{r}}\left\|\mathbf{Y}_{j}-\sqrt{\frac{E}{N_{t}}} \mathbf{S}_{D} \mathbf{H}_{j}\right\|^{2} .
$$

\section{A. Prior works: low complexity front-end \\ III. Lte-Advanced Receiver Architecture}

The analog complexity issue concerning advanced receiver using digital processing has been very little addressed [2]. For both multi-antenna solutions and multi-band allocations, the performance gain implies an increase of the complexity and of the consumption of the analog front-end. Therefore the previous studies aim to improve the performance-consumption-complexity trade-off offered by the analog front-end architectures dedicated to the new radio techniques. Two independent studies explore the use of a single common front-end for the processing of signals received by several antennas: - The double IQ architecture dedicated to the simultaneous reception of two separate frequency band signals, - The code multiplexing architecture for the multi-antenna reception.

As mentioned in Sec. 2, the future LTE-Advanced standard takes in consideration a discontinuous spectrum usage, as well as multi-antenna techniques for the downlink transmission. In order to answer to these specifications the actual state of the art of radiofrequency receivers imposes the use of dedicated frontend stackup architectures. For example, if considers a dual-band discontinuous spectrum scenario where two antennas are used for the reception of a $2 \times 2$ MIMO transmission, the receiver will have to integer a four front-end stack-up. Each of these front-ends is dedicated to the processing of one of the contributions obtained from the combination of two antennas and two non-adjacent frequency bands. It becomes obvious that this method imposes high complexity, but especially high power consumption.

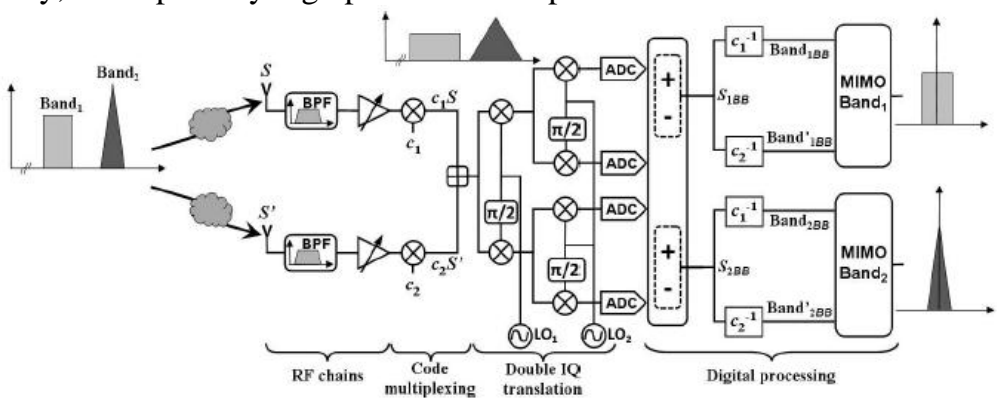

Figure-2 Architecture LTE-Advanced Receivers capable of realizing a multi-antennas processing of a discontinuous spectrum signal.

Based on the dual band simultaneous reception and the RF code multiplexing architectures, proposing unique frontend architecture dedicated to the LTE-Advanced receivers. In this paper, chosen process of two antennas reception generalized for several antennas by increasing the length of the codes.

\section{B. Structure introduction}

The proposed structure is shown in Fig. 2. It consists of 4 main parts: the RF dedicated chains, the RF code multiplexing, the double IQ structure and the digital part including code de-multiplexing and multi-antenna processing. Consider that the two input signals $\mathrm{S}$ and $\mathrm{S}$ ' are the result of the propagation of a bi-band signal through two different channels. Let Band1-Band2 and Band'1-Band'2 are the two pairs of contribution composing $\mathrm{S}$ and $\mathrm{S}$ ' respectively, such as:

$$
\begin{gathered}
\mathrm{S}=\text { Band1 + Band2, (1) } \\
\mathrm{S}^{\prime}=\text { Band'1 + Band'2. (2) }
\end{gathered}
$$

Once received, each of the two input signals are separately filtered and amplified by a dedicated RF filter and a dedicated LNA, as shown in Fig. 2. 
The multiplexing of the four contributions is realized by a two-step method. Firstly, the RF orthogonal code spreading technique used in order to multiplex the two input signal as described in [5]. Let c1S, c2S' be the signals resulting from the coding technique:

$$
\begin{aligned}
\mathrm{c} 1 \mathrm{~S} & =\mathrm{c} 1 \mathrm{Band} 1+\mathrm{c} 1 \text { Band2 } \\
\mathrm{c} 2 \mathrm{~S}^{\prime} & =\mathrm{c} 2 \text { Band' } 1 \text { + c2Band'2. }
\end{aligned}
$$

The orthogonal codes must have a chip time two times smaller than the symbol time of each of the two bands. When multiplied with the orthogonal codes, each of the frequency bands Band1 and Band2 of the signal c1S (respectively Band'1 and Band'2 for the signal c2S') will be spread in the same manner around their own central frequency, as shown in Fig. 2. This multiplexing step is concluded by the addition of the c1S and c2S' signals. Therefore, by using the code spreading technique, the antennas' contributions having the same central frequency are multiplexed two by two. The second block of the architecture assessed is implementing the double IQ technique. While this technique is generally used in image frequency rejection front-ends, recently proposed a novel architecture dedicated to the simultaneous reception of two separate frequency band signals [3]. The double IQ structure multiplexes the two bands during the translation from RF to baseband; it consists of two IQ translations as described in Fig. 2. An important aspect is the choice of the first local oscillator LO1 frequency. This frequency is chosen in such a manner that each of the useful signals has its spectrum situated in the image frequency band of the other. In the baseband domain, the four obtained signals are digitized. The baseband component of the two useful signals is obtained by using two dedicated basic operations processing described. Theses digital outputs S1BB and S2BB are the baseband contribution of the two coded frequency bands:

$$
\begin{aligned}
& \mathrm{S} 1 \mathrm{BB}=\mathrm{c} 1 \mathrm{~B} \text { and1BB }+\mathrm{c} 2 \mathrm{Band} \text { ' } 1 \mathrm{BB},(5) \\
& \mathrm{S} 2 \mathrm{BB}=\mathrm{c} 1 \mathrm{Band} 2 \mathrm{BB}+\mathrm{c} 2 \mathrm{Band} 2 \mathrm{BB} .(6)
\end{aligned}
$$

In order to de-multiplex each of these two pairs of signals, applied two digital filters matched to the codes. Once separately obtain the two pairs of signals corresponding to the two antennas reception of a dual band signal, two antennas SIMO processing is used, one for each band. The antenna diversity digital processing used in this study is the classical Sample Matrix Inversion (SMI) [12]. It uses the training sequence in order to compute the optimal combination of different signals using an adaptive algorithm based on a Minimum Mean Square Error (MMSE) criterion.

\section{Simulation Results}

The physical layer requirements concerning LTE-Advanced are not yet finalized. A particular RF signal model utilized for these simulations. In order to obtain an OFDM signal having a discontinuous spectrum, a signal composed of the addition of two 802.11g non overlapping channels is used. The transmission channel is an AWGN (Additive White Gaussian Noise) one. The results presented in Fig. 4 show the BER evolution for different Eb-No of the antenna input signal. The receivers are using either the proposed unique front-end architecture ("Double Structure") or the dedicated front-end stack-up architecture ("Stack-up"). The results shown in Fig. 4 are mainly concerning the SIMO receivers using the two different architectures when receiving the dual-channel signal. Meanwhile, also the BER evolution shows that the receivers are realizing a SISO reception. Both in the SIMO and SISO case, the performance obtained when using the front-end stack-up architecture are slightly better compare to those obtained with the unique front-end structure. This is due to the fact that the codes used by the proposed structure are not perfectly orthogonal during simulation. It observe that the SIMO reception offers the same performance gain when using the two architectures, it turns around $3 \mathrm{~dB}$. This processing gain is the theoretical result that a two antennas system should reach in an AWGN channel. At conclusion considers that the performance of the proposed architecture is the same as those of the dedicated front-end stack-up.

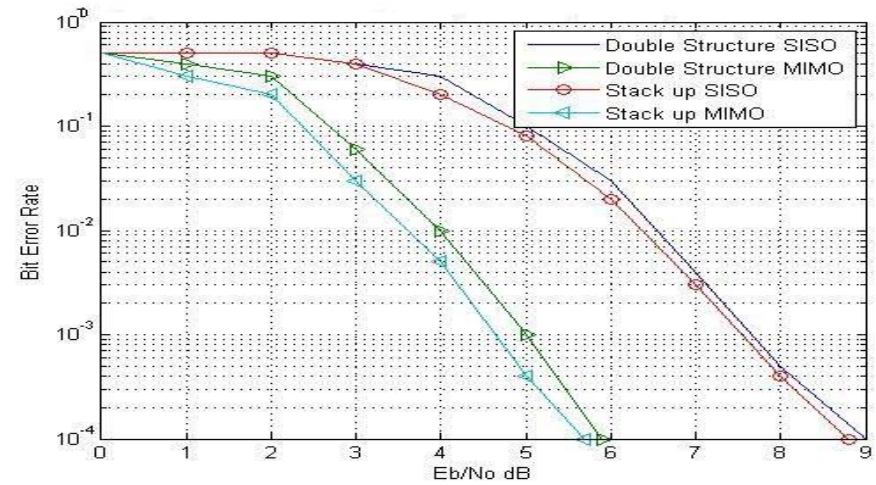

Figure No 3:- Simulated BER evolutions during the simultaneous SIMO reception Of a signal composed of two non-overlapping 802.11g channels. 


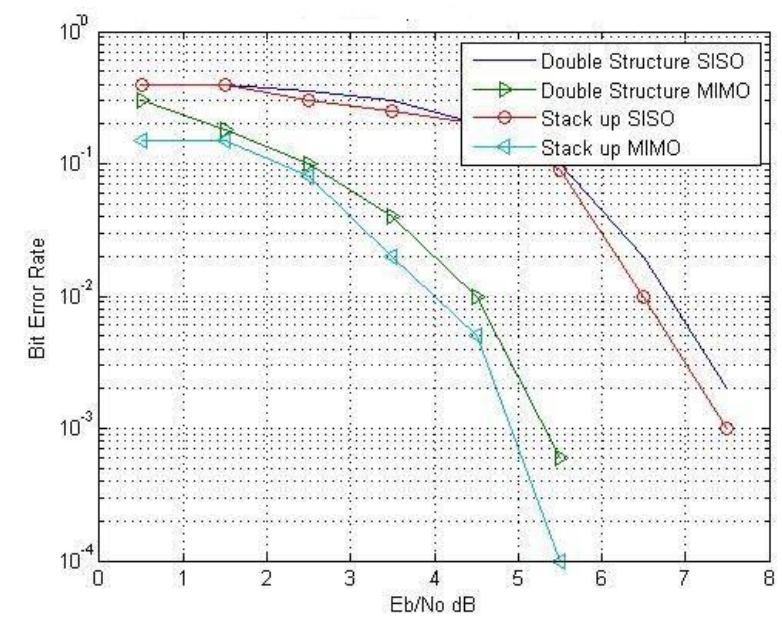

Figure No 4:- Measured BER evolutions during the simultaneous SIMO reception

Of a signal composed of two non-overlapping $802.11 \mathrm{~g}$ channels.

In the previous figures ( 3 and 4 ) the simulation has been proved the MIMO system and SISO with two different architectures. The results of them shown that MIMO with two different architectures (stack-up and double structures) are much better than SISO with its two architecture. The figure 5 shows the enchantment on the two systems (MIMO and SISO) with their two architectures. The enhancement has been done by combined the multiband MIMO system with the UWB model. The results in the figure 5 viewed the BER evolution for different Eb/No for multiband UWB-MIMO system compared with MIMO (stack-up and double structures) and SISO (stack-up and double structure). It observed that the multiband UWB-MIMO reception offers better performance gain than using two architectures (stack-up and double structures) through MIMO or SISO models.

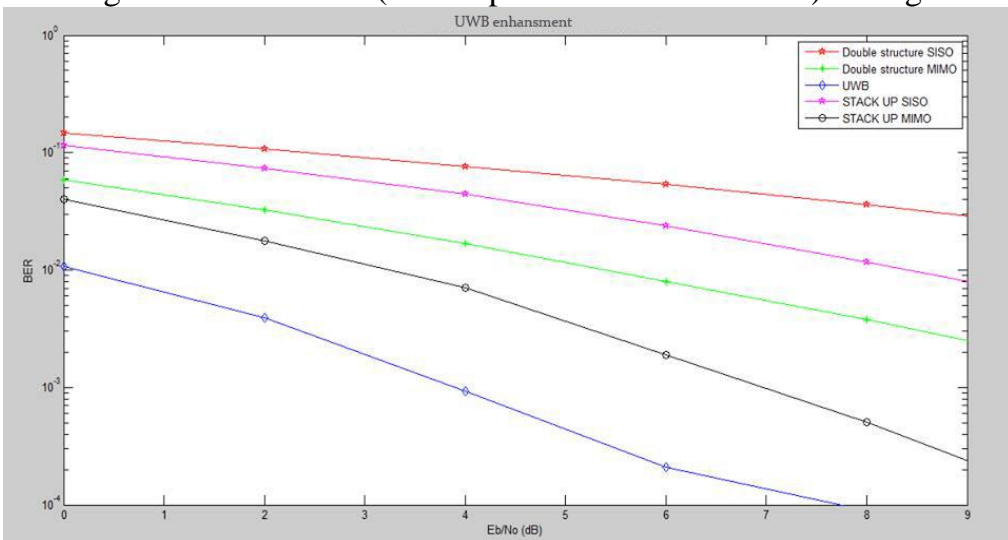

Figure No 5:- Measured BER evolutions during the simultaneous Multiband UWB-MIMO reception With MIMO and SISO (Stack-up and Double structure).

\section{CONCLUSION}

In this paper, proposed a multiband MIMO coding framework for UWB systems. By a technique of band hopping in combination with jointly coding across spatial, temporal and frequency domains, the proposed scheme is able to exploit all available spatial and multipath diversities, richly inherent in UWB environments. From the theoretical results, could draw some interesting conclusions as follows. First, the effect of Nakagami$\mathrm{M}$ fading parameter on the diversity gain is insignificant, and the diversity advantages obtained in Nakagami-M fading and Rayleigh fading channels are almost the same. In this paper, novel low complexity architecture was presented. This type of architecture is a good candidate to the integration in the receivers dedicated to the future LTE-Advanced standard. One of the major advantages of this architecture is its low complexity and therefore low power consumption. Indeed, despite the use of a de-multiplex block in the digital domain, the proposed architecture offers a significant $33 \%$ of power gain compared with the solution proposed by the actual state of the art. 


\section{REFERENCES}

[1] Revision of Part 15 of the Commission's Rules Regarding Ultra-Wideband Transmission Systems, First Report and Order. Fed.Commun.Comm. Rep., FCC, Washington DC, ET-Docket 98-153.

[2] R. A. Scholtz, "Multiple access with time-hopping impulse modulation, "in Proc. MILCOM Conf., Boston, MA, Oct. 1993, pp. 447-450.

[3] M. Z. Win and R. A. Scholtz, "Impulse radio: How it works," IEEE Commun. Lett., vol. 2, no. 2, pp. 36-38, Feb. 1998.

[4] M. L.Welborn, "System considerations for ultra-wideband wireless networks, "in IEEE Radio Wireless Conf., Aug. 2001, pp. 5-8.

[5] E. Saberinia and A. H. Tewfik, "Pulsed and nonplused OFDM ultra wideband wireless personal area networks," in IEEE Conf. Ultra Wideband Systems Tech., Nov. 2003, pp. 275-279.

[6] A. Batraet al., "Design of a multiband OFDM system for realistic UWB channel environments," IEEE Trans. Microw. Theory Tech., vol. 52, no.9, pp. 2123-2138, Sep. 2004.

[7] www.3gpp.org.

[8] R. Van Nee and R. Prasad, OFDM for Wireless Multimedia Communications, Artech House, 2000.

[9] T. Kaiser, A. Bourdoux, H. Boche, J. Rodriguez Fonollosa, J. BachAndersen, and W. Utschick, Smart Antennas: State of the Art, EurasipBook Series on Signal Processing \& Communications, 2005.

[10] I. Burciu, G. Villemaud, J. Verdier, and M. Gautier, "A 802.11g and UMTS Simultaneous Reception Front-End Architecture using a double IQ structure," in Proceedings of IEEE Vehicular Technology Conference(VTC09-Spring), April 2009.

[11] I. Burciu, M. Gautier, G. Villemaud, and J. Verdier, "Method for Processing Two Signals Received by a Single Terminal," International Patent WO/2010/031944, Mars 2010.

[12] M. Gautier and G. Villemaud, "Low complexity antenna diversity frontend: Use of code multiplexing," in Proceedings of IEEE Wireless Communication and Networking Conference (WCNC09), April 2009.

[13] M. Gautier, I. Burciu, and G. Villemaud, "New antenna diversity frontend using code multiplexing," in Proceedings of European Conference on Antennas and Propagation (EuCAP09), March 2009. 